\title{
Impacto del marketing digital a las empresas colombianas emergentes
}

\author{
Remedios Catalina Pitre Redondo* \\ Suleica Elvira Builes Zapata* \\ Hugo Gaspar Hernández Palma***
}

Fecha de recibido: 15 de mayo de 2020

Fecha de aprobado: 20 de septiembre de 2020

\begin{abstract}
Para citar este artículo: Pitre Redondo, R. C., Builes Zapata, S. E., \& Hernández Palma,
H. G. (2021). Impacto del marketing digital a las empresas colombianas emergentes. Revista Universidad \& Empresa, 23(40), 1-20. https://doi.org/10.12804/revistas.urosario.
\end{abstract}

edu.co/empresa/a.9114

\section{Resumen}

El marketing digital cobra cada vez mayor importancia en los entornos competitivos de los mercados globales; en este sentido, el comprador de hoy cuenta con características puntuales derivadas de su adopción de las nuevas tecnologías, que a su vez lo llevan a ejercer una toma de decisiones con un mayor análisis de información. De ello surge el interés por observar las condiciones de incorporación de las estrategias de marketing digital en las empresas colombianas y los aportes que pueden generar para su desarrollo. Por lo anterior, se llevó a cabo una revisión documental, bajo un enfoque cualitativo y descriptivo, que permitió detallar los principales aportes del marketing digital en el mundo, sus herramientas y estrategias y las condiciones colombianas para

* Economista, Universidad del Magdalena (Colombia). Magíster en Gestión y Dirección de Empresas Sociales, Universidad Simón Bolívar (Colombia). Docente de Trabajo Social, Universidad de La Guajira (Colombia). Correo electrónico: rpitre@ guajira.edu.co

** Administradora de empresas, Universidad de La Guajira (Colombia). Magíster en Educación, RUDE, Universidad de La Guajira. Docente de la Facultad Educación, Universidad de La Guajira. Correo electrónico: suleicabuiles@uniguajira.edu.co

*** Ingeniero Industrial, Universidad Libre (Colombia). Especialista en Estudios Pedagógicos, Universidad cuc (Colombia). Especialista en Diseño y Evaluación de Proyectos, Universidad Autónoma del Caribe (Colombia). Magíster en Sistemas de Gestión, Universidad Autónoma del Caribe. Docente del Programa de Administración de Empresas, Universidad del Atlántico (Colombia). Correo electrónico: hugoghernandezpalma@gmail.com. ORCID: https://orcid. org/0000-0002-3873-0530 
su aplicación. Como conclusiones destacadas se evaluaron los principales componentes del marketing digital, las condiciones de accesibilidad de las TIC en la población nacional y sus tendencias de consumo a través del comercio electrónico, además de las dificultades y barreras culturales que deben ser superadas por las pymes para su adecuado aprovechamiento. Lo anterior para terminar con un análisis de los aportes relacionados con competitividad, internacionalización de mercados, innovación, sostenibilidad económica e interacción con los clientes.

Palabras clave: barreras digitales; comercio electrónico; innovación; marketing digital.

\title{
Impact of Digital Marketing on Emerging Colombian Companies
}

\begin{abstract}
Digital marketing is becoming increasingly important in the competitive environments of global markets. In this sense, today's buyer has specific characteristics derived from his/her adoption of new technologies, which in turn lead him/her to make decisions with greater analysis of information, from which arises the interest in observing the conditions of incorporation of digital marketing strategies in Colombian companies and the contributions they can generate for their development. In accordance with this, a documentary review was carried out under a qualitative and descriptive approach, which allowed detailing the main contributions of digital marketing in the world, its tools and strategies, and the Colombian conditions for its application. We evaluated the main components of digital marketing, the conditions of accessibility to ICTs in the national population, and their consumption trends through electronic commerce in addition to the difficulties and cultural barriers that must be overcome by smes for their proper use. We finished with an analysis of the contributions related to competitiveness, internationalization of markets, innovation, economic sustainability, interaction with customers, and customer relationship.
\end{abstract}

Keywords: Digital barriers; e-commerce; innovation; digital marketing.

\section{Impacto do marketing digital nas empresas colombianas emergentes}

\section{Resumo}

O marketing digital está cada vez mais importante nos ambientes competitivos dos mercados globais; nesse sentido, o comprador de hoje possui características pontuais que são decorrentes da adoção de novas tecnologias, que uma vez, o levam a tomar decisões baseadas em uma maior análise de informação; de onde surge o interesse de observar as condições de incorporação das estratégias de marketing digital nas empresas colombianas, e as contribuições que as mesmas podem gerar para seu desenvolvimento. Diante do exposto, realizamos uma revisão documental, com um enfoque qualitativo e descritivo, que permitiu detalhar as principais contribuições do marketing digital no mundo, suas ferramentas e estratégias, e as condições colombianas para sua aplicação. Como conclusões destacadas, se avaliaram os principais componentes do marketing digital, as condições de acessibilidade das NTIC na população nacional e sus tendências de consumo por meio do comércio eletrônico; além das dificuldades e barreiras culturais que devem ser superadas pelas PMEs para seu adequado aproveitamento; para finalmente concluir com uma análise das contribuições relacionadas com a competitividade, internacionalização de mercados, inovação, sustentabilidade econômica e a interação com os clientes.

Palavras-chave: barreiras digitais; comércio eletrônico; inovação; marketing digital. 


\section{Introducción}

La competitividad y el objetivo de alcanzar un desarrollo organizacional más eficiente se han convertido en motor de innovación y de búsqueda de nuevas alternativas de gestión que permitan incrementar la eficacia de sus acciones y la mejora de sus resultados (Franco Restrepo et al., 2014). En este sentido, el marketing digital ha surgido como respuesta a la nueva tendencia tecnológica que rodea a la población global y se ha convertido en una herramienta indispensable para alcanzar mercados mayores, en cantidad y cobertura, con la disponibilidad de canales de comunicación con los clientes reales y potenciales, que permitan mayor interacción e inmediatez (Cantillo Guerrero, 2013).

El comprador de hoy día cuenta con nuevas herramientas, en especial de comunicación, lo que lo hace más exigente en el momento de tomar sus decisiones (Valencia et al., 2014). En relación con esto, el usuario actual usa las novedades tecnológicas para adquirir mayor información y comparar productos, en especial los que se encuentran disponibles en internet; además, se comunica constantemente con otros individuos de la web, intercambiando opiniones y evaluaciones de los productos o servicios que pretende (Kotler et al., 2018). Por otra parte, en los casos en los que las ventas se concretan en las tiendas físicas, los usuarios también han explorado sus otras alternativas a través de la red, por lo que llegan al sitio plenos de información, con la cual comparan lo que están percibiendo directamente y ello les permite una mejor evaluación sobre su decisión de compra (Restrepo Torres \& Parra Osorio, 2013).

En la actualidad, internet se encuentra en su versión web 5.0, basada en el reconocimiento de las emociones, el cual es el resultado de la evolución dada a partir de su nacimiento, que inicia desde la resolución de operaciones básicas (web 0.0), siguiendo con el papel de información a través de páginas web (web 1.0), continuando con la aparición de redes sociales y comunidades virtuales (web 2.0), seguida por el uso de la inteligencia artificial (web 3.0) y finalizando en su etapa inmediatamente anterior, con el uso de mejoras en la conectividad, uso de espacios tridimensionales, computación en la nube, entre otras características (web 4.0) (Foroudi et al., 2017; Khanzode \& Sarode, 2016). De esta manera, así como internet ha ido creciendo en su funcionalidad, del mismo modo lo ha hecho su 
funcionalidad, trayendo aportes al mercado que resultan significativos en el momento de establecer las ventajas competitivas para su sostenibilidad (Figueroa González, 2015).

Las cifras del comercio electrónico han venido en crecimiento a través de los años. Así es como, de acuerdo con Soler Patiño (2014), para el 2010, este tipo de ventas alcanzaba alrededor del 13\% de las transacciones mundiales; así mismo, se estima que para el 2020, este concepto alcance el $17 \%$ de todas las ventas del sector retail; y para el 2050 el impacto sea mucho más significativo. Por otra parte, en Colombia se observó un valor estimado de 1000 millones de dólares durante el 2011, cifra que llegaría a los 5200 millones de dólares en el 2016.

En virtud de lo expuesto, este artículo de revisión evalúa los principales aspectos que dan importancia al marketing digital, su oportunidad de desarrollo en Colombia y los beneficios que conlleva su incorporación en las estrategias comerciales de las empresas nacionales.

\section{Metodología}

El estudio desarrollado en esta publicación se basó en los lineamientos de la revisión documental, a partir de una búsqueda de artículos científicos relacionados con el tema de investigación, de publicación reciente en bases de datos como Scielo, Emerald, Elsevier, Springer, entre otros; a su vez complementados con datos estadísticos de orden oficial e información gubernamental, los cuales se revisaron con un enfoque descriptivo, con el fin de trazar un retrato del contexto nacional e internacional del tema de análisis, y de esta manera decantar los resultados de mayor relevancia (Chohan, 2019; Lambert \& Lambert, 2012). 


\section{Resultados}

\subsection{El marketing digital}

En relación con el uso que la población mundial hace de la tecnología, el marketing digital se ha convertido en una alternativa, cada vez más atractiva de comercialización, basada en el empleo de recursos tecnológicos y medios digitales para el desarrollo de estrategias fundamentadas en el establecimiento de comunicación directa con el cliente, a fin de lograr la venta de productos o servicios, el posicionamiento de marcas o la fidelización a ellas (Castro Gómez et al., 2017).

El marketing digital hace parte de una estrategia comercial que pretende incrementar las cifras de ventas de una compañía, y de acuerdo con esto, algunas organizaciones deciden subcontratar todos o algunos de los aspectos que intervienen en su desarrollo. Así, existen organizaciones que deciden administrar todo el proceso, desde el manejo de sus tecnologías de la información y comunicación (TIC) hasta el contacto final con el cliente, pasando por el empleo del recurso humano especializado en este tipo de tareas; mientras que otras deciden subcontratar todas las etapas, dejando en manos de proveedores especializados la responsabilidad comercial de sus empresas (Prada, 2016). En cualquier caso, la toma de decisión debe tomar en consideración todas las responsabilidades inherentes con cada estilo de trabajo, sabiendo que el objetivo final redunda en una mejora de la comunicación, interacción con los clientes y en el incremento de las cifras de ventas de sus productos o servicios (Järvinen \& Karjaluoto, 2015). Los teóricos que han profundizado en este tipo de estrategias han logrado desglosar sus principales aportes de acuerdo con lo mostrado en la figura 1. 


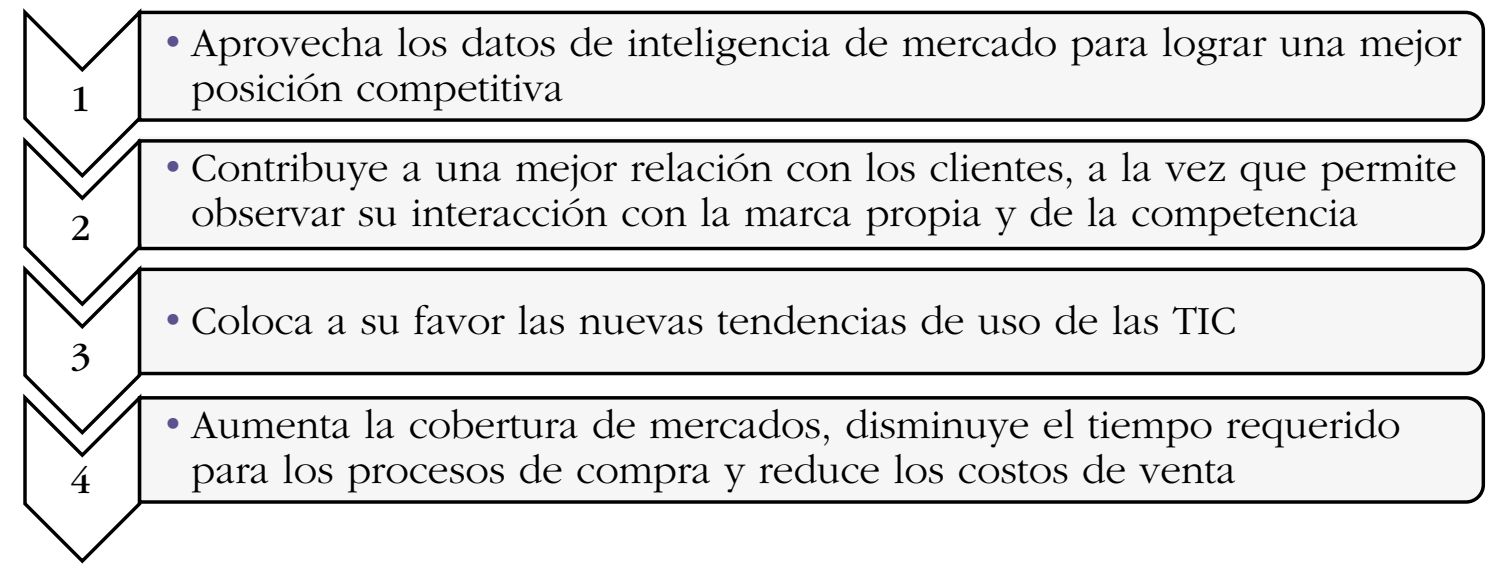

Figura 1. Principales aportes del marketing digital

Fuente: elaboración propia con base en Teixeira et al. (2017) y Chaffey y Ellis (2019).

De acuerdo con el contexto analizado, en estas nuevas estrategias de mercadeo intervienen nuevos actores; dicho de otro modo, diversos tipos de consumidores digitales influyen en la comunicación y toma de decisión de los compradores. En este sentido, la Cámara de Comercio de Bogotá (2019) ha identificado los siguientes tipos como se puede ver en la figura 2:

\begin{tabular}{|l|}
\hline \multicolumn{1}{|c|}{ Prosumer } \\
\hline - Genera contenidos \\
para la marca, en \\
torno a opiniones y \\
experiencias en la \\
página web de la \\
empresa, en sus \\
redes sociales o \\
foros \\
\end{tabular}
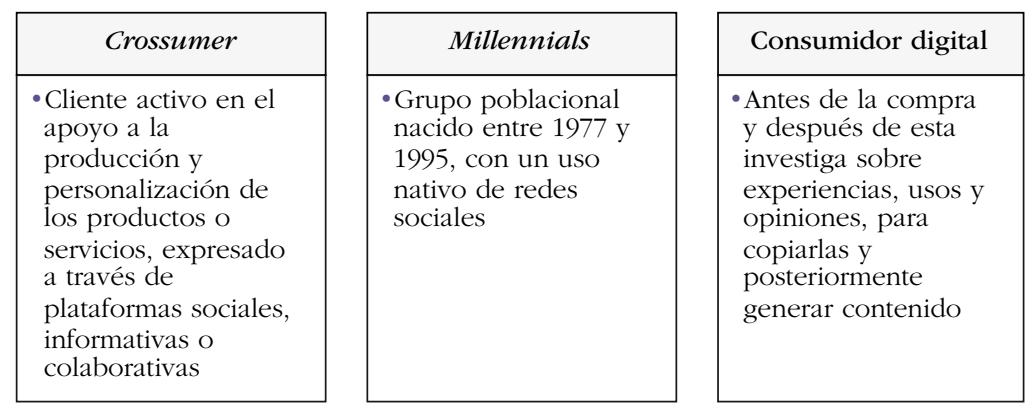

Figura 2. Tipos de consumidores digitales

Fuente: elaboración propia con base en Cámara de Comercio de Bogotá (2019) y Apolo Buenaño et al. (2015).

Así mismo, las herramientas y técnicas útiles al marketing digital son muy variadas y su empleo dependerá de las necesidades y recursos disponibles para la inversión por parte de la empresa interesada. Se destacan:

- Página web: se trata de la construcción de una página en línea, donde el cliente puede encontrar información relevante de la empresa y los productos 
o servicios que ofrece. Ello facilita un canal de contacto y, a la vez, suministra contenido que contribuye a la toma de decisión de compra a favor de la compañía. Para que este sea un elemento de comercialización realmente útil, se debe vigilar su estructura, composición, contenidos y accesibilidad (Frick, 2013).

- Tiendas en línea: elemento consistente en una página web en la cual el cliente puede adquirir sus productos mediante herramientas seguras de compra digital. En estas se deben tener en cuenta la incorporación de aspectos como búsquedas en catálogo, detalle de los productos, ofertas y novedades, así como plataformas de pago seguro. Dichas tiendas pueden ser de propiedad exclusiva de la empresa o compartida en plataformas web especializadas (Chaffey \& Smith, 2013).

- Search Engine Optimization (SEO): técnica encargada de mejorar las páginas web de determinada empresa para que sea fácilmente encontrada por los motores de búsqueda, a partir de la utilización de ciertas palabras clave, para lo cual se deben tener en cuenta las páginas en las que aparecen, el número de búsquedas al mes y otros portales que pudieran estar asociados (Ortega Fernández, 2015).

- Search Engine Marketing (SEM): consiste en contratar publicidad o anuncios de pago en los motores de búsqueda, de tal manera que dichos administradores reproduzcan estos en las páginas web de los clientes potenciales, de acuerdo con su perfil de navegación e intereses (Ramdani et al., 2013).

- Content marketing: basado en la generación de contenidos, asociados con el producto o servicio que se pretende comercializar, y de interés relevante para el cliente potencial; distribuido a través de redes sociales, videos, infografías, tutoriales, libros, guías, entre otros (Taiminen \& Karjaluoto, 2015).

- Social media: consiste en el uso de aplicaciones web para la creación y publicación de contenido digital; de esta manera, las empresas están en capacidad de circular su publicidad, a la vez que recibe retroalimentación constante de la 
receptividad de sus contenidos y las tendencias de navegación de su público objetivo; además de los comportamientos de sus competidores y proveedores (Simula et al., 2015).

- Email marketing: o mercadeo por suscripción. Como su nombre lo indica, se trata del envío de correos electrónicos con información exclusiva de productos, ofertas o promociones que se encuentren enlazados a páginas web que permitan ampliar la información suministrada. Para esto, resulta necesario que los remitentes se hayan suscrito al servicio de noticias y novedades, con el fin de que esta estrategia no se convierta en una molestia para el cliente potencial (Soininen, 2015).

Con base en lo expuesto, vale la pena resaltar que las técnicas y herramientas de marketing digital no solo tienen que ver con la publicación de contenido atractivo para los clientes potenciales, sino además su utilidad depende de la capacidad de la organización para desarrollar análisis cuantitativos y cualitativos de los datos asociados con el comportamiento de consumo de sus clientes, y de esta manera poder crear y llevar a cabo estrategias comerciales realmente efectivas para los intereses de venta de la compañía (Iturralde, 2016; Narváez Vásquez \& Montalvo Escamilla, 2014).

\subsection{El acceso a la tecnología y el comercio electrónico en Colombia}

Para el desarrollo de las estrategias de marketing digital resulta indispensable estudiar las condiciones de acceso y uso de la tecnología de la población que se pretende impactar. Así es como, en relación con lo anterior, Londoño Arredondo et al. (2018) destacan algunas de las barreras más relevantes para el acceso de la tecnología en el mundo, que definen la brecha digital: por ejemplo, la dificultad de acceso que tienen algunas poblaciones de carácter nacional se encuentran originadas por las características generacionales, nivel educativo y socioeconómico o el tipo de infraestructura de las comunicaciones instaladas en el país. Al respecto de esto, un estudio del Departamento Administrativo Nacional de Estadística (DANE, 2017) estableció que, para el 2016, solo el $58.1 \%$ de los colombianos tuvo acceso a internet (distribuidos según la figura 3) y, adicionalmente, apenas el $45.2 \%$ contaba con un computador personal en casa. 


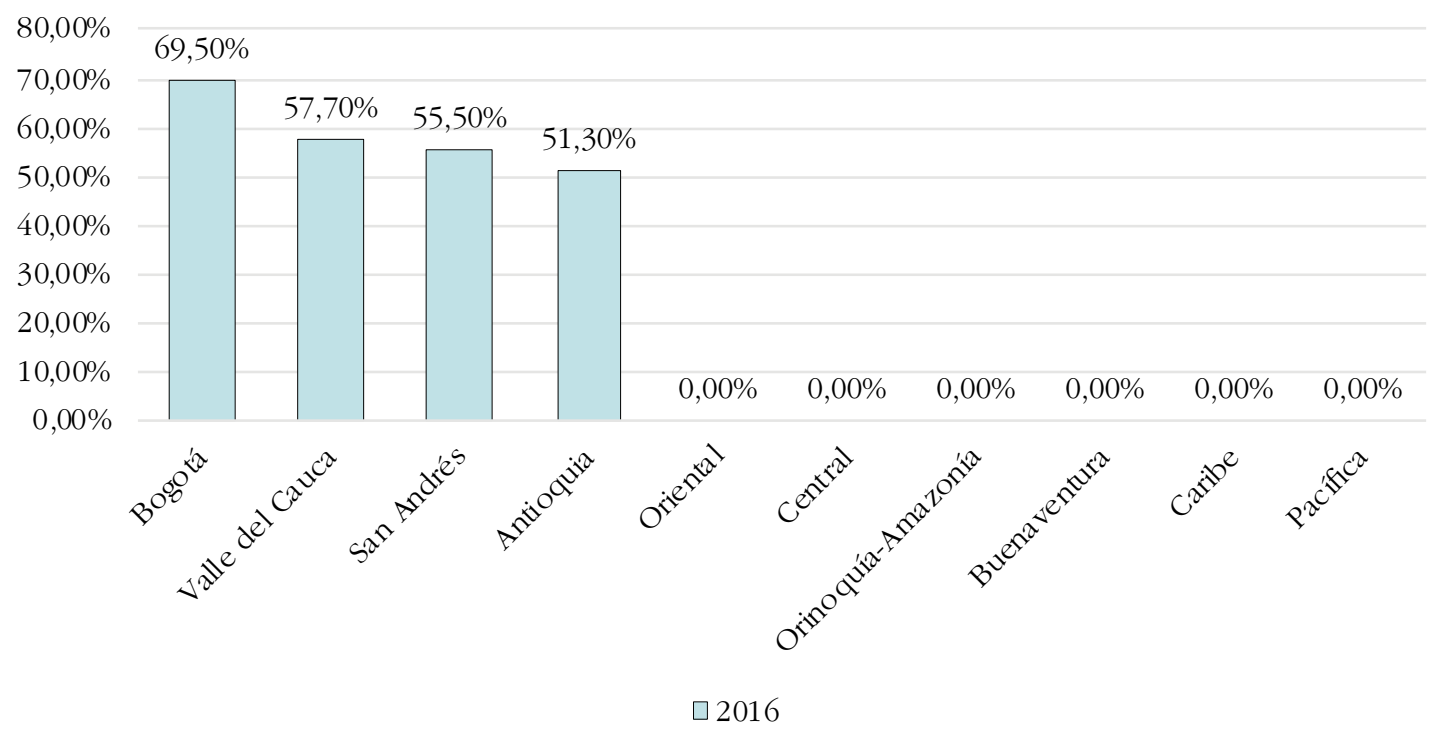

Figura 3. Distribución del acceso a internet en Colombia (\%)

Fuente: elaboración propia con base en DANE (2017).

En relación con los datos representados en la figura 3, es importante tener en cuenta que, en la actualidad, las pymes colombianas se inclinan a desarrollar sus estrategias de marketing digital a través de las redes sociales, como Facebook, Instagram, Twitter o You'Tube, en las cuales la primera se lleva la mayor participación, con un $25 \%$ de presencia de empresas colombianas, ya que esta plataforma ofrece un $70 \%$ de conectividad de sus potenciales clientes (Torres et al., 2017). De acuerdo con lo anterior, en la figura 4 se presenta el porcentaje de uso de comercio electrónico de las empresas colombianas, por sector.

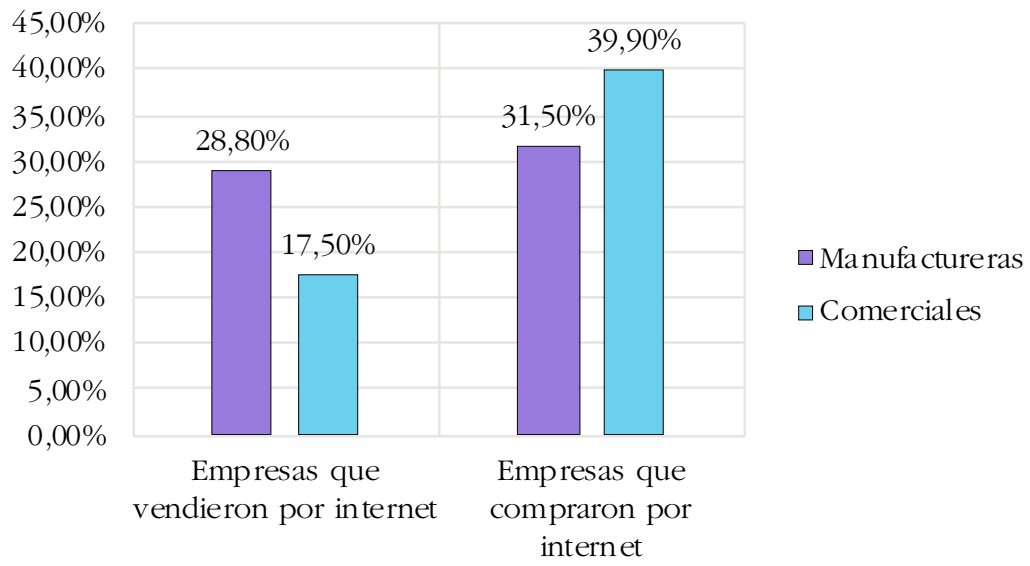

Figura 4. Uso del comercio electrónico en las pymes colombianas, en el 2017 (\%) Fuente: elaboración propia a partir de DANE (2018). 
De esta forma, en relación con la figura 4, llama la atención la diferencia de porcentaje de empresas comerciales que compraron más por internet versus lo que vendieron, cuando la relación debería ser contraria. Adicionalmente, en cuanto al sector servicios, las empresas dedicadas al alojamiento vendieron sus servicios en un $38.9 \%$, seguidas por las agencias de viajes (33.5\%) y la educación superior privada (22.5\%); mientras que aquellas enfocadas en el desarrollo de sistemas informáticos fueron las que más compraron (27.4\%), seguidas por las agencias de viajes (21.7\%), servicios de alojamiento (21.2\%) y la educación superior privada (20.5\%) (figura 5). Lo anterior puede ser explicado en razón a que estos tipos de servicios son aquellos que cuentan con plataformas digitales más desarrolladas alrededor del mundo (DANE, 2018).

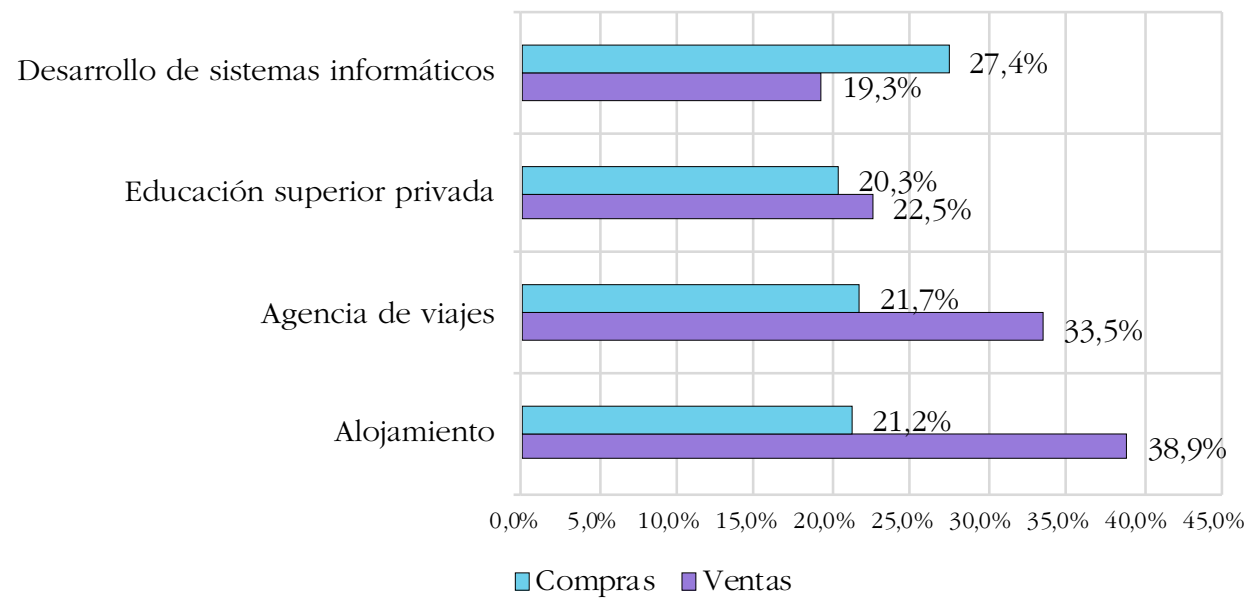

Figura 5. Porcentaje de uso del comercio electrónico en las pymes del sector servicio en el 2017 Fuente: elaboración propia a partir de DANE (2018).

Lo anterior lo sustentan estudios estatales que han encontrado que, en Colombia, los productos que más se compran en internet tienen que ver con el entretenimiento, el turismo y el esparcimiento; seguidos por la tecnología y la moda, venta motivada principalmente por los aspectos de seguridad, la logística de envío y el servicio posventa (Striedinger Meléndez, 2018).

Por su parte, Castellanos Galeano et al. (2016) también han destacado los resultados expuestos en el informe de la Red Design Systems, donde se muestra que los colombianos usan internet, en su mayoría (69\%), para el acceso y visualización de fotos y videos; el 54\% lo usa para encontrar información sobre empresas, y el $41 \%$ lo emplea para la búsqueda de opiniones, experiencias o tendencias de compras de productos. Así mismo, Sanabria Díaz etal. (2016) resaltan que Colombia es el cuarto país en Latinoamérica con 
el mayor número de usuarios de internet (55.9\%), precedido de Argentina (67\%), Chile (59\%) y Uruguay (57\%).

Si bien es cierto que el comercio electrónico es cada vez más representativo en el mundo, también es importante tener en cuenta que no todos los compradores potenciales dominan las tecnologías a cabalidad, situación que puede representar un obstáculo que debería vencer el marketing digital (Hernández y Hernández et al., 2014). En consecuencia, se debe asegurar una comunicación asertiva con los clientes a través de diversos canales de interacción, de tal manera que el usuario reconozca a la empresa y sus contenidos digitales con mayor confianza para incentivar el mayor número de transacciones para la adquisición de dichos productos o servicios (Mazzarol, 2015).

Por otra parte, es igualmente necesario resaltar la existencia de barreras culturales relacionadas con el uso de las tecnologías y el marketing en las empresas colombianas, sobre todo en las mipymes, las cuales conforman en mayor porcentaje del aparato productivo del país (Mora et al., 2015). En este sentido, Diago Ortiz y Martínez Tobar (2017) establecen que cinco de cada diez empresas utilizan las TIC e internet para desarrollar sus estrategias de mercadeo y publicidad. En relación con lo anterior, se tiene la idea que los postulados teóricos alrededor de las estrategias corporativas se han desarrollado para grandes empresas; adicionalmente, no se cuenta con una adecuada comprensión de los conceptos, al pretender utilizarlos de manera estandarizada, como si todos los contextos empresariales fueran iguales, y persiste la tendencia a pensar que lo extranjero es mejor. De ahí que las empresas se inclinen a copiar los recursos utilizados en el campo internacional, sin tener en cuenta el verdadero contexto nacional (Ortiz Morales et al., 2016; Páramo, 2015).

\subsection{Beneficios del uso del marketing digital en las empresas colombianas}

Internet representa un elemento de gran ventaja para las empresas colombianas, por cuanto ofrece una ventana al mundo que facilita una mejor interacción con los clientes, así como el conocer características, novedades y tendencias del mercado en el que se desenvuelve y por el mismo medio darse a conocer con la inversión de menores recursos de tiempo y capital (Gutiérrez-Leefmans \& Nava-Rogel, 2016). De esta forma, internet les permite a las pymes posicionarse en el mercado a la par con las grandes empresas, ofreciendo sus productos 
y servicios de una forma más efectiva y facilitando su acceso a mercados que otrora eran inaccesibles (Cepeda Palacio et al., 2017).

De la mano con lo anterior, los procesos comerciales desarrollados con apoyo de internet logran una mayor venta de productos y una mejor interacción con los clientes y proveedores, a través del uso de herramientas especializadas para el Customer Relationship Management (CRM), que a su vez optimizan la atención comercial y ofrecen una mayor accesibilidad y disponibilidad de flujos de información (Sainz de Vicuña, 2018). Adicionalmente, otro de los beneficios obtenidos a partir de la incorporación del marketing digital en las estrategias comerciales es la innovación, tomando en consideración que su principal insumo es la adquisición y generación de conocimiento desarrollado a partir de diversas fuentes de información. De acuerdo con lo anterior, en la figura 6 se presentan los hallazgos de Bernal-Torres y Frost-González (2015).

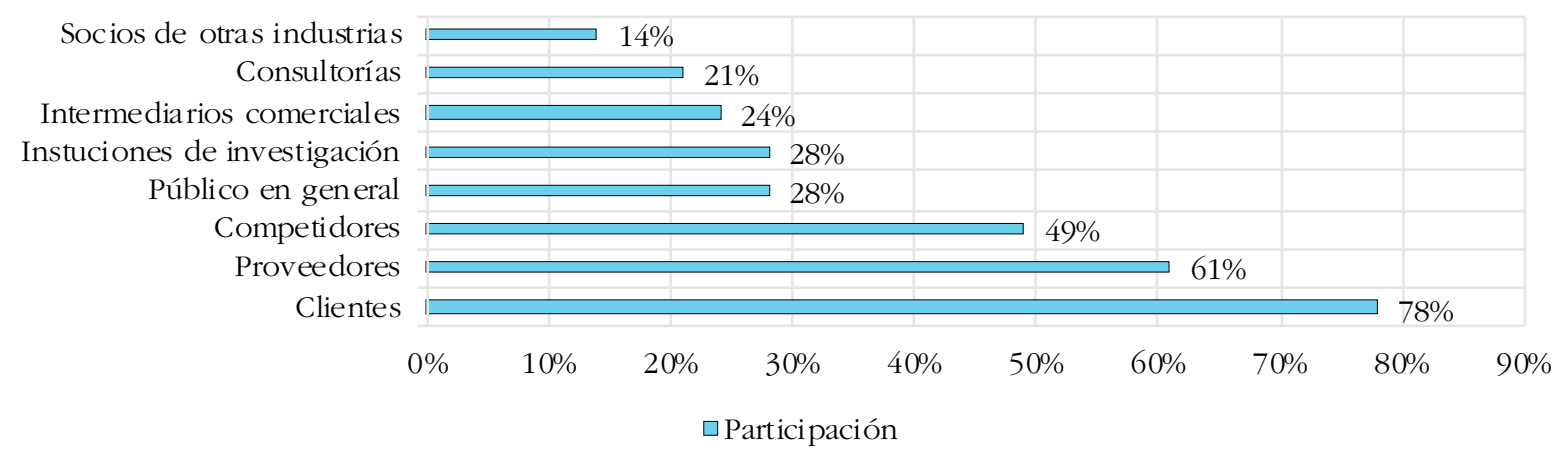

Figura 6. Principales fuentes de información utilizadas para la innovación Fuente: elaboración propia con base en Bernal-Torres y Frost-González (2015).

Como se observa en la figura 6, las cuatro principales fuentes de información corresponden a clientes, proveedores, competidores y público en general, por lo cual la evaluación de su comportamiento y el análisis de los datos (componentes fundamentales del marketing digital) se convierte en parte esencial de los procesos de innovación (Scuotto et al., 2017). Entonces, sin estas herramientas, la evolución de los productos y servicios se hace cada vez más difícil y regazada, y ello perjudica la competitividad de las empresas colombianas (Hernández-Fuentes \& Sánchez-Mojica, 2017).

Desde otro punto de vista, el uso de herramientas y estrategias de marketing digital ejerce un rol fundamental en los propósitos de internacionalización de las empresas colombianas, 
que a su vez se combinan con otros factores, como el entorno de la organización, las características de los directivos, la construcción de redes, las capacidades empresariales y otros determinantes de mercadeo. Así, un buen empleo de las ventajas competitivas que ofrece este elemento aporta buenas oportunidades de éxito en las estrategias de exportación y la expansión de mercados (Escandón Barbosa \& Hurtado Ayala, 2014).

En cuanto al impacto financiero, el estudio de Novoa et al. (2016) proporciona una visión de sus principales aportes. De acuerdo con esto, los autores realizaron un estudio econométrico, haciendo la salvedad que dichos resultados se ven afectados por la manera como las empresas usan estas tecnologías y el tipo de estrategias que utilizan. Dentro sus principales resultados destacan que un $1 \%$ de inversión en medios digitales genera un aumento en la rentabilidad financiera del $0.3807 \%$; además de un incremento del $0.0536 \%$ en la relación pasivo-ventas.

Por otra parte, los beneficios del marketing digital no solo provienen de su uso en el interior de las empresas; su empleo en las entidades gubernamentales proporciona entornos de mercado más favorables y con mayores oportunidades de desarrollo para sus actores (Marland et al., 2017). Ejemplo de lo anterior se observa en la estrategia de City Marketing, que utiliza las herramientas tecnológicas para ampliar su espectro de cobertura y, de esta manera, identificar y establecer los principales intereses y necesidades de los públicos reales o potenciales que hacen parte de una comunidad o que la visitan para su aprovechamiento y disfrute, de tal manera que se puedan crear alternativas de negocio que logren satisfacer dicha demanda. Dicho concepto se ve redituado especialmente en los sectores de turismo y comercio, los cuales se ven impactando por un mayor flujo de personas atraídas por la oferta de servicios de determinada ciudad o población (Andrade Yejas, 2016).

\section{Discusión}

Los resultados obtenidos en el presente estudio pueden contrastarse con investigaciones como la realizada por Pitre Redondo et al. (2018), en la cual se logró determinar el gran 
efecto que tiene el uso del marketing digital como medio de crecimiento y desarrollo de nuevos emprendimientos en Colombia en el sector de los calzados. En este caso, los autores proponen que el marketing digital se ha convertido en la apuesta más relevante para los microemprendimientos del país, que por los altos costos y bajos resultados de los medios tradicionales acuden a este como una solución práctica.

Si bien es cierto que el marketing digital se caracteriza por los bajos costos y su alta efectividad, se debe destacar que este también es aprovechado por empresas de más alta envergadura, pues se ha convertido en el medio de difusión más relevante de los últimos años, tanto que ha superado con creces a medios como la televisión y la radio. Un basamento importante para esta afirmación no es solo nuestro estudio, también se puede citar a Chiquillo Rodelo et al. (2018), quienes consideraron que los procesos de marketing empresariales han sufrido importantes cambios a raíz de las nuevas tendencias, las cuales se inclinan hacia las redes sociales como pilar fundamental. Esto ciertamente cobra vital importancia en las empresas emergentes, las cuales no se ven en la necesidad de adaptarse a los cambios recientes, pues se gestan dentro del entorno y son parte de ellos, por lo que tienen una relación mucho más recíproca entre colocarse en el ámbito competitivo y proponer nuevos paradigmas (Brito Carillo et al., 2018).

\section{Conclusiones}

Los entornos competitivos del mundo globalizado obligan a las empresas a mejorar su estrategia de innovación y gestión para lograr resultados más eficientes, eficaces y productivos. En este sentido, el marketing digital surge como una herramienta de desarrollo que permite acercar a la empresa hacia su público objetivo, así como trabajar sobre información real de sus comportamientos de consumo que facilite el desarrollo de mejoras en sus productos y servicios.

Ahora bien, internet ha venido evolucionando desde que se creó para resolver problemas de operaciones básicas hasta la actualidad, cuando busca el reconocimiento de las emociones para desarrollar una mejor oferta. Allí es donde las empresas colombianas 
-en especial las pymes - deben basar sus estrategias para el aprovechamiento de dichos recursos en favor de su propia sostenibilidad.

En línea con lo expuesto, también fue posible observar el crecimiento del comercio electrónico en el mundo, pues cada vez es más relevante para las empresas que desean lograr el crecimiento deseado. Así es como los aportes del marketing digital pueden ser muy variados, y del mismo modo las herramientas y técnicas para su incorporación en las operaciones corporativas; sin embargo, su utilidad se ve condicionada por los tipos de solución aportadas a las necesidades puntuales de la organización que la persigue, y al nivel de inversión destinada para su desarrollo.

En este sentido, como compilación de los hallazgos observados, el uso de estas estrategias ayuda a las empresas colombianas a establecer una mejor interacción con sus clientes y consumidores, a la vez que obtiene retroalimentación valiosa para su mejora continua; adicionalmente, la utilización de internet facilita extender mercados hacia territorios lejanos, y la internacionalización, sin incurrir en costos de riesgo significativo. Por otra parte, la disponibilidad de software y aplicaciones especializadas en la red contribuye al desarrollo de una mejor atención comercial, con mayor accesibilidad y mejores flujos de información.

Todo lo anterior, aplicado de manera estratégica, lleva a mejoras tanto de innovación como de competitividad y, por consiguiente, a rendimientos económicos, aspectos que pueden ser reproducidos en la administración estatal, mediante el uso del marketing digital en el desarrollo de estrategias que fortalezcan la atracción de mercados extranjeros, que a su vez aporten capital de inversión para el desarrollo de la sociedad colombiana.

\section{Referencias}

Andrade Yejas, D. A. (2016). Estrategias de marketing digital en la promoción de Marca Ciudad. Revista EAN (80), 59-72. https://doi.org/10.21158/01208160.n80.2016.1457

Apolo Buenaño, D., Altamirano Barriga, M. V., Vásconez Cadena, V. E., \& Cevallos Darquea, M. I. (2015). Usuarios, clientes y consumidores digitales: Consideraciones para su abordaje 
desde el marketing y la comunicación corporativa. Redmarka: Revista de Marketing Aplicado, (14), 3-19. https://doi.org/10.17979/redma.2015.01.014.4879

Bernal-Torres, C. A., \& Frost-González, S. (2015). Innovación abierta en empresas colombianas: Reto a superar. Revista Venezolana de Gerencia, 20(70), 252-267. https://doi.org/10.31876/ revista.v20i70.19996

Brito Carillo, J., Hernández P., H., \& Pitre Redondo, R. (2018). Decision making under the multicriteria approach to identify marketing mix strategies. Contemporary Engineering Sciences, 11(52), 2581-2589. https://doi.org/10.12988/ces.2018.86271

Cámara de Comercio de Bogotá. (2019, 16 de noviembre). Estrategias de éxito en marketing digital para el sector turismo. https://bibliotecadigital.ccb.org.co/bitstream/handle/11520/23176/ESTRATEGIAS\%20DE\%20\%C3\%89XITO\%20EN\%20MARKETING\%20 DIGITAL.pdf?sequence=1\&isAllowed=y

Cantillo Guerrero, E. (2013). Factores claves en la gestión de mercadeo que inciden en la competitividad del sector de comunicaciones gráficas de la ciudad de Barranquilla, Colombia. Revista EAN, (75), 140-151. https://doi.org/10.21158/01208160.n75.2013.776

Castellanos Galeano, J. F., Loaiza, M. H., \& Cuesta Iglesias, C. A. (2016). Importancia de las TIC para la competitividad de las pymes en Colombia. Puente, 10(1), 93-99. https://doi. org/10.18566/puente.v10n1.a10

Castro Gómez, B., Esteban Jiménez, S., Moreno Toro, J., \& Saavedra López, A. (2017). Planeamiento estratégico del marketing digital en Colombia [tesis de maestría]. Pontificia Universidad Católica del Perú. http://tesis.pucp.edu.pe/repositorio/bitstream/handle/20.500.12404/8792/CASTRO_ESTEBAN_PLANEAMIENTO_DIGITAL_COLOMBIA.pd$\mathrm{f}$ ? sequence $=3 \&$ isAllowed $=\mathrm{y}$

Cepeda Palacio, S. D., Velásquez Estrada, L. J., \& Marín Gómez, B. E. (2017). Análisis evaluativo a los procesos de marketing en la internacionalización de las pequeñas y medianas empresas de alimentos de Medellín. Estudios Gerenciales, 33(144), 271-280. https://doi. org/10.1016/j.estger.2017.06.006

Chaffey, D., \& Ellis-Chadwick, F. (2019). Digital marketing. Pearson uk.

Chaffey, D., \& Smith, P. R. (2013). eMarketing eXcellence: Planning and optimizing your digital marketing. Routledge.

Chiquillo Rodello, J., Pitre Redondo, R., \& Hernández Palma, H. (2018). Business marketing and its transformation with the insertion of social networks. Indian Journal of Science and Technology, 11(25). https://doi.org/10.17485/ijst/2018/v11i25/129095

Chohan, U. W. (2019). Documentary research: Positing innovations in a national budget process. https://doi.org/10.4135/9781526469489 
Departamento Administrativo Nacional de Estadística (DANE). (2017, 16 de marzo). Encuesta Nacional de Calidad de Vida-ECV 2016. https://www.dane.gov.co/files/investigaciones/ condiciones_vida/calidad_vida/Boletin_Tecnico_ECV_2016.pdf

Departamento Administrativo Nacional de Estadística (DANE). (2018, 28 de diciembre). Indicadores básicos de tenencia y uso de tecnologías de la información y comunicación en 2017. https://www.dane.gov.co/files/investigaciones/boletines/tic/bol_empresas_2017.pdf

Diago Ortiz, A., \& Martínez Tobar, M. (2017). Elementos para el desarrollo de una estrategia de marketing digital que permita mejorar la gestión de marketing en empresas agroindustriales en el departamento del Cauca. Administración \& Desarrollo, 47(2), 184-193. https://doi.org/10.22431/25005227.364

Escandón Barbosa, D. M., \& Hurtado Ayala, A. (2014). Los determinantes de la orientación exportadora y los resultados en las pymes exportadoras en Colombia. Estudios Gerenciales, 30(133), 430-440. https://doi.org/10.1016/j.estger.2014.05.002

Figueroa González, E. G. (2015). Rentabilidad y uso de comercio electrónico en las micro, pequeñas y medianas empresas del sector comercial. RIDE. Revista Iberoamericana para la Investigación y el Desarrollo Educativo, 6(11). https://doi.org/10.23913/ride.v6i11.177

Foroudi, P., Gupta, S., Nazarian, A., \& Duda, M. (2017). Digital technology and marketing management capability: achieving growth in smes. Qualitative Market Research: An International Journal, 20(2), 230-246. https://doi.org/10.1108/qmr-01-2017-0014

Franco Restrepo, J. G., Restrepo Restrepo, J. C., \& Sánchez Giraldo, J. C. (2014). La gestión del mercadeo: Un aporte a la competitividad de las pequeñas empresas del sector servicios en Medellín. Pensamiento E Gestión, (37), 150-174. https://doi.org/10.14482/pege.37.7025

Frick, T. (2013). Return on engagement: Content, strategy and design techniques for digital marketing. Routledge.

Gutiérrez-Leefmans, C., \& Nava-Rogel, R. M. (2016). Mercadotecnia digital y las pequeñas y medianas empresas: revisión de la literatura. Enlace: Revista Venezolana de Información, Tecnología y Conocimiento, 13(1), 45-61. https://www.redalyc.org/pdf/823/82346016004.pdf

Hernández y Hernández, D., Ramírez-Martinell, A., \& Cassany, D. (2014). Categorizando a los usuarios de sistemas digitales. Pixel-Bit. Revista de Medios y Educación, (44), 113-126. https://doi.org/10.12795/pixelbit.2014.i44.08

Hernández-Fuentes, S., \& Sánchez-Mojica, K. Y. (2017). Innovación y competitividad: micro y pequeñas empresas del sector agroindustrial en Cúcuta. Revista de Investigación, Desarrollo e Innovación, 8(1), 23-33. https://doi.org/10.19053/20278306.v8.n1.2017.7368

Iturralde, M. F. (2016). Content marketing: Innovación e información a través de las redes sociales. Revista de Investigación Sigma, 2(1). https://doi.org/10.24133/sigma.v2i1.925 
Järvinen, J., \& Karjaluoto, H. (2015). The use of web analytics for digital marketing performance measurement. Industrial Marketing Management, (50), 117-127. https://doi.org/10.1016/j. indmarman.2015.04.009

Khanzode, C. A., \& Sarode, R. (2016). Evolution of the world wide web: from web 1.0 to 6.0. International journal of Digital Library Services, 6(2), 1-11. http://www.ijodls.in/uploads/3/6/0/3/3603729/ijodls121.pdf

Kotler, P., Kartajaya, H., \& Setiawan, I. (2018). Marketing 4.0: Transforma tu estrategia para atraer al consumidor digital. LID.

Lambert, V. A., \& Lambert, C. E. (2012). Qualitative descriptive research: An acceptable design. Pacific Rim International Journal of Nursing Research, 16(4), 255-256. https://www. tci-thaijo.org/index.php/PRIJNR/article/download/5805/5064/

Londoño Arredondo, S., Mora Gutiérrez, Y. J., \& Valencia Cárdenas, M. (2018). Modelos estadísticos sobre la eficacia del marketing digital. Revista EAN, (84), 167-186. https://journal. universidadean.edu.co/index.php/Revista/article/view/1923/1740

Marland, A., Lewis, J. P., \& Flanagan, T. (2017). Governance in the age of digital media and branding. Governance, 30(1), 125-141. https://doi.org/10.1111/gove.12194

Mazzarol, T. (2015). sMEs engagement with e-commerce, e-business and e-marketing. Small Enterprise Research, 22(1), 79-90. https://doi.org/10.1080/13215906.2015.1018400

Mora, E. H., Vera, M. A., \& Melgarejo, Z. A. (2015). Planificación estratégica y niveles de competitividad de las mipymes del sector comercio en Bogotá. Estudios Gerenciales, 31(134), 79-87. https://doi.org/10.1016/j.estger.2014.08.001

Narváez Vásquez, G. A., \& Montalvo Escamilla, E. (2014). Best practice in the use of social networks marketing strategy as in sMes. Procedia-Social and Behavioral Sciences, 148(25), 533-542. https://doi.org/10.1016/j.sbspro.2014.07.076

Novoa, A., Sabogal, M., \& Vargas, C. (2016). Estimación de las relaciones entre la inversión en medios digitales y las variables financieras de la empresa: Una aproximación para Colombia. Revista EAN, (80), 12-25. https://doi.org/10.21158/01208160.n80.2016.1313

Ortega Fernández, E. (2015). seo: Clave para el crecimiento de las pymes. Opción, 31(6), 652675. https://www.redalyc.org/pdf/310/31045571040.pdf

Ortiz Morales, M. D., Joyanes Aguilar, L. J., \& Giraldo Marín, L. M. (2016). Los desafíos del marketing en la era del big data. e-Ciencias de la Información, 6(1), 1-31. https://doi. org/10.15517/eci.v6i1.19005

Páramo, D. (2015). Marketing en Colombia. Pensamiento E Gestión, (38), 7-10. http://dx.doi. org/10.14482/pege.37.7018 
Pitre Redondo, R., Manjarrés de Ávila, W., \& Hernández Palma, H. (2018). Digital marketing as a promoter of entrepreneurship in the footwear sector in Colombia. Contemporary Engineering Sciences, 11(82), 4059-4067. https://doi.org/10.12988/ces.2018.88469

Prada, R. (2016). Las Tic, factor de efectividad en la aplicación del mercadeo digital en negocios de retail. Revista EAN, (80), 105-116. https://doi.org/10.21158/01208160.n80.2016.1460

Ramdani, B., Chevers, D., \& A. Williams, D. (2013). smes adoption of enterprise applications: A technology-organisation-environment model.Journal of Small Business and Enterprise Development, 20(4), 735-753. https://doi.org/10.1108/jsbed-12-2011-0035

Restrepo Torres, M. L., \& Parra Osorio, J. F. (2013). La gestión de clientes: Exploración sobre la práctica entre un grupo de profesionales de mercadeo en Colombia. Cuadernos de Administración, 26(46), 83-106. https://doi.org/10.11144/Javeriana.cao26-46.gces

Sainz de Vicuña, J. (2018). El plan de marketing digital en la práctica. EsIc.

Sanabria Díaz, V. L., Torres Ramírez, L. A., \& López Posada, L. M. (2016). Comercio electrónico y nivel de ventas en las mipymes del sector comercio, industria y servicios de Ibagué. Revista EAN, (80), 132-154. https://journal.universidadean.edu.co/index.php/Revista/article/view/1463/1416

Scuotto, V., Del Giudice, M., \& Carayannis, E. G. (2017). The effect of social networking sites and absorptive capacity on SMEs' innovation performance. The Journal of Technology Transfer, 42(2), 409-424. https://doi.org/10.1007/s10961-016-9517-0

Simula, H., Töllmen, A., \& Karjaluoto, H. (2015). Facilitating innovations and value co-creation in industrial B2B firms by combining digital marketing, social media and crowdsourcing. En Marketing dynamism \& sustainability: Things change, things stay the same... (pp. 254263). Springer. https://doi.org/10.1007/978-3-319-10912-1_84

Soininen, N. (2015). Improving SMEs brand awareness by digital marketing: A guide for sMEs. Turku University of Applied Sciences. https://core.ac.uk/download/pdf/38126144.pdf

Soler Patiño, A. (2014). ¿Hacia dónde va el comercio electrónico en Colombia? Ploutos, 4(1), 17-24. https://journal.ean.edu.co/index.php/plou/article/view/1371/1331

Striedinger Meléndez, M. P. (2018). El marketing digital transforma la gestión de pymes en Colombia. Cuadernos Latinoamericanos de Administración, 14(27). https://doi. org/10.18270/cuaderlam.v14i27.2652

Taiminen, H. M., \& Karjaluoto, H. (2015). The usage of digital marketing channels in sMEs. Journal of Small Business and Enterprise Development, 22(4), 633-651. https://doi. org/10.1108/jsbed-05-2013-0073

Teixeira, S., Martins, J., Branco, F., Gonçalves, R., Au-Yong-Oliveira, M., \& Moreira, F. (2017, octubre). A theoretical analysis of digital marketing adoption by startups. En 
International Conference on Software Process Improvement (pp. 94-105). Springer. https:// doi.org/10.1007/978-3-319-69341-5_9

Torres, R., Rivera, J., Cabarcas, R., \& Castro, Y. (2017). La efectividad del uso del marketing digital como estrategia para el posicionamiento de las pymes para el sector comercio en la ciudad de Barranquilla. Investigación y Desarrollo en TIC, 8(2), 3-5. https://doi. org/10.15359/ri.90-1.3

Valencia, A., Palacios, I., Cedeño, J., \& Collins, N. (2014). Influencia del marketing digital en el proceso de decisión de compra. Revista Científica y Tecnológica UPSE, 2(1), 1-5. https:// doi.org/10.26423/rctu.v2i1.38 